BONPLANDIA 20(2). 2011

ISSN: 0524-0476

\title{
MIELES Y PLANTAS EN LA MEDICINA CRIOLLA DEL NORTE DE MISIONES, ARGENTINA
}

\author{
FERNANDO ZAMUDIOํㅛ \& NORMA I. HILGERT ${ }^{1}$
}

Summary: Zamudio, F. \& N. I. Hilgert. 2011. Honeys and plants used in the traditional medicine of northern Misiones, Argentina. Bonplandia 20(2): 165-184.

The uses of wild bees products and plants in the traditional medicine of the province of Misiones, Argentina, was studied; particularly regarding bee species and therapeutic uses of honey-based concoctions. In this paper, 412 different uses were reported, comprising 9 wild bees taxa. Among the useful products collected from wild bee hives, honey was the most frequently used; mainly for respiratory afflictions and as functional foods. Honeys are used as the basic ingredient of 61 concoctions, where 39 plant species, 6 manufactured products, and 5 products of animal origin may be mixed-in alternatively. Consensus between informants was found regarding primary products, but not in the combinations used in the recipes. Honeys produced by Tetragonisca fiebrigi and Apis mellifera stand out due to their widespread use and high value given by inhabitants. These honeys could be considered as complementary therapeutic products, but a priori not as interchangeable ones. However, specificities in the medicinal use of Tetragonisca fiebrigi and Apis mellifera honeys should be understood as a continuum, shaped by other factors such as availability and access.

Keywords: Atlantic Forest, Ethnobiology, medicinal mixtures, Tetragonisca fiebrigi, Apis mellifera.

Resumen: Zamudio, F. \& N. I. Hilgert. 2011. Mieles y plantas en la medicina criolla del norte de Misiones, Argentina. Bonplandia 20(2): 165-184.

Se analizan los usos medicinales de los productos obtenidos de colonias de abejas silvestres, en cuanto al uso diferencial de estos recursos según las especies de abejas y el consenso de usos de mezclas que involucran mieles. Se reportaron 412 usos correspondientes a 9 taxones de abejas. Entre los productos de los nidos de estos insectos, la miel es el más utilizado como remedio, principalmente para el sistema respiratorio y como alimento funcional. La misma se emplea en diversas formas de preparación y están presentes en 61 preparados mixtos donde se combinan con plantas (39 especies), productos manufacturados (6) y de origen animal (5). Se halló consenso en el uso de algunos productos, no así en las combinaciones en las que intervienen. Las mieles de Tetragonisca fiebrigi y de Apis mellifera se destacan por su importancia y versatilidad de uso. Éstas pueden ser consideradas recursos medicinales complementarios y a priori no intercambiables. Sin embargo, las especificidades deben ser entendidas como alternativas no categóricas, cuyo uso es moldeado por otros factores como disponibilidad y acceso.

Palabras clave: Bosque Atlántico, Etnobiología, mezclas medicinales, Tetragonisca fiebrigi, Apis mellifera.

${ }^{1}$ Consejo Nacional de Investigaciones Científicas y Técnicas (CONICET). Centro de Investigaciones del Bosque Atlántico (CeIBA). Instituto de Biología Subtropical (IBS). Facultad de Ciencias Forestales, Universidad Nacional de Misiones. Andresito 21, 3370-Puerto Iguazú, Misiones, Argentina. E- mails: zamufer@yahoo.com.ar; normahilgert@yahoo.com.ar 


\section{Introducción}

La necesaria parcialización de la información propia del reduccionismo moderado (es decir reducir, teniendo en cuenta la variedad, la emergencia y conservando un contexto amplio de análisis), combinada con la integración epistémica (es decir, aquella que requiere la intervención de varias disciplinas), es un camino adecuado para el avance de la ciencia como un todo, según algunos autores (Bunge, 1983; Mahner \& Bunge, 2000). En la etnobiología, la heterogeneidad teóricometodológica, el pluralismo epistemológico de sus enfoques, así como sus bases conceptuales, filosóficas y metodológicas suponen un desafío para el análisis de temas tan complejos como la interrelación entre las sociedades humanas, la cultura y la naturaleza (Alexiades, 2010; Bandeira, 2010). Uno de los aspectos más valiosos de esta disciplina es el análisis integrador de las culturas, sus valores y su entorno (Ladio, 2010); aunque, inevitablemente, la reducción y la integración conllevan a exponer con mayor detalle, o a ponderar algunos aspectos en detrimento de otros.

Los estudios etnobiológicos, en general, han categorizado conceptualmente la información local desde una perspectiva etic, donde los recursos animales o vegetales utilizados son estudiados de forma separada (Hanazaki \& al., 2006, Martínez-Crovetto, 1968). En el mismo sentido, al analizar los usos medicinales y alimentarios, surgen conflictos para incluir aquellos recursos que, desde la perspectiva emic, se emplean como recursos preventivos (es decir, alimentos que evitan enfermedades). Respecto a estos alimentos medicinales, se ha propuesto categorizarlos como alimentos funcionales y analizarlos contemplando la superposición de estos dos universos de uso (Etkin, 1994; Etkin \& Ross, 1982; Pieroni \& Quave, 2006).

Por otra parte, el estudio de los sistemas superpuestos de alimentación, prevención y cura suponen una mayor complejidad cuando se contemplan las prácticas que combinan productos de diferente origen. En efecto, las mezclas constituyen una forma común de preparación de remedios entre diversas culturas (Ngokwey, 1995; Ríos Ruiz \& Martínez Francés, 2003; Vandebroek \& al., 2010). Si se revisa la literatura se encuentran muchos ejemplos donde se ofrecen datos sobre estos preparados pero, alternativamente se pierden detalles. Según se trate de estudios etnobotánicos u etnozoológicos se suele dar mayor información sobre los productos de origen vegetal y animal respectivamente. La miel por ejemplo, suele ser un producto "gris" entre estas categorías, que en algunos casos se la cita como una entidad en sí misma sin ofrecer detalles de las especies de las que provienen (Hilgert \& Gil, 2006; Scarpa, 2004) y, por el contrario, en otros se menciona su uso en combinación con plantas sin precisar su identidad taxonómica (Alves \& Rosa, 2007; Modro \& al., 2009).

En el contexto latinoamericano, donde la miel es un producto obtenido de una gran diversidad de abejas y avispas silvestres (Hymenoptera: Apidae y Vespidae), además de la naturalizada abeja europea Apis mellifera (Silveira \& al., 2002), el análisis etnobiológico de este recurso es un tema de difícil inclusión en esas clásicas agrupaciones mencionadas. Los insectos productores de miel cuentan con representaciones en diferentes ámbitos culturales de comunidades indígenas y criollas (Costa-Neto, 1998; Falchetti \& Nates Parra, 2002; Posey, 1983a, b). En algunas culturas, incluida la occidental, la miel es considerada un producto de origen animal; en cambio, en otras, como es el caso de los Mby'a-guaraní, se encuentra dentro del dominio del mundo vegetal (Cebolla, 2009).

En el presente aporte se analiza el uso medicinal de los productos obtenidos de las colonias de abejas entre los pobladores rurales del norte de la provincia de Misiones. Se analiza el uso diferencial de estos recursos según las especies productoras. Se describen las formas de preparación, administración y dolencias tratadas con mieles y/o productos preparados a base de miel. Por otra parte, siguiendo la propuesta de estudios recientes en los que se hace un minucioso análisis de las mezclas empleadas con fines medicinales o profilácticos, tales como las denominadas garrafadas en Brasil 
(Ngokwey, 1995), los "herberos" en la región alicantina de la península Ibérica (Ríos Ruiz \& Martínez Francés, 2003) y las "botellas" en la República Dominicana (Vandebroek $\&$ al., 2010), se analizan distintos aspectos de los usos medicinales de las mezclas que involucran el uso de mieles preparadas en el norte de Misiones.

Las preguntas que guían estos análisis son: ¿el uso de las mieles varía según la especie que las produce? ¿se emplean para el tratamiento o la profilaxis de dolencias similares? Es decir, ¿hay mieles que pueden usarse de modo indistinto para algunas dolencias? En relación al uso de las mieles en preparados compuestos por diferentes productos, se analiza: ¿cuáles son los recursos (plantas u otros productos) más utilizados y cómo se combinan? ¿hay consenso en el uso de estas combinaciones en la población local?

Las mieles de abejas sin aguijón son un recurso empleado en casi todo el mundo con fines alimenticios y medicinales (Ahmad \& al., 2003; Mahawar \& Jaroli, 2007; Meda \& al., 2004; Schwarz, 1948). En América hay registros de su aprovechamiento desde antes de la llegada de los españoles (Medrano \& Rosso, 2009; Schwarz, 1948) hasta la actualidad (Arenas, 2003; Costa-Neto, 2002; Costa-Neto \& Motta, 2010; Hill \& al., 1984; Modro \& al., 2009; Vit \& al., 2004; Zamudio $\&$ al., 2010).

La miel, larvas y polen constituyen fuentes valiosas de alimentos y medicinas para grupos indígenas y criollos (Arenas, 2003; Cadogan, 1957; Costa-Neto, 2002; Costa-Neto \& Oliveira, 2000; Hill \& al., 1984; NogueiraNeto, 1997; Posey, 1983a; Quezada-Euán \& al., 2001;). Las ceras (cerumen) obtenidas de sus colonias son un elemento importante para la confección de herramientas como pegamento para arcos y flechas (Posey, 1983a) y, antiguamente, en la confección de moldes para forjar piezas de oro (Falchetti \& Nates Parra, 2002; Schwarz, 1948). Para los Ache de Paraguay, la miel de diferentes especies de himenópteros, incluidas las abejas sin aguijón, es un producto importante en su dieta, en especial, en períodos de escasez de otros recursos (Hill \& al., 1984).

En la literatura se encontró que la miel de meliponinas es usada en América para dolencias y enfermedades que abarcan los sistemas respiratorio (resfriados, catarro, tos, afecciones pulmonares, asma), dérmico (heridas, quemaduras, acné, mordeduras de perros o serpientes, micosis), gástrico (constipación, diarrea, úlcera), reproductivo (impotencia), urinario (pus en orina), ocular (infecciones oculares, ptérigion) y óseomuscular (reumatismo, dolores musculares). En estos casos, la miel se administra vía oral (té, jarabe), en forma tópica (heridas, quemaduras), sobre el pecho en cataplasmas, y se inhala el humo de la quema de cerumen (mezcla de cera con resinas vegetales) o de diferentes partes del nido (Costa-Neto, 2002). Según Schwarz (1948) estos usos son coincidentes con los de las abejas sin aguijón de África.

Se encontró que la miel utilizada con fines medicinales se administra de forma pura o en mezclas con diferentes especies de plantas y otros productos como huevo, aceite, grasa, sangre y leche, entre otros (Costa-Neto, 2002; Hernández Cano \& Volpato, 2004; Schwarz, 1948).

En la Argentina, conocemos poco sobre la diversidad de usos de la miel entre los pobladores rurales e indígenas. En la literatura hay referencias sobre sus usos medicinales, generados en el marco de estudios más amplios (dentro de estudios etnobotánicos y/o etnofarmacológicos), por lo que hay información poco precisa sobre las mieles y su rol en los preparados donde se las incluye (Arias Toledo \& al., 2007; Martínez, 2008; Martínez \& al., 2004; Scarpa, 2004). Arenas (2003) analiza minuciosamente el uso alimentario de mieles de himenópteros entre Tobas y Wichi del Chaco central de la Argentina, menciona los usos medicinales de las mieles pero, dado el objeto del estudio, no brinda especificaciones. Del mismo modo, Cebolla (2009) y Rodrigues (2005) desarrollan la importancia y concepción de la miel entre los Mby'a-guaraní de Misiones y del sur de Brasil, respectivamente. Cebolla (2009) brinda datos sobre el uso de las mieles entre los que se indican algunos usos medicinales aunque éste no es un tema central en su obra. 


\section{Materiales y Métodos}

\section{Área de estudio y actores involucrados}

Se trabajó en el Departamento Gral. Manuel Belgrano, al norte de la Provincia de Misiones (Argentina). El área limita al este con el Departamento Iguazú y con los estados brasileños de Río Grande do Sul y Santa Catarina, al norte y noroeste respectivamente (Fig. 1). Desde el punto de vista biogeográfico, el área corresponde al Bosque Atlántico del Alto Paraná; el que constituye la porción más austral y occidental del complejo de eco-regiones del Bosque Atlántico y la que mantiene la mayor extensión de bosques remanentes (Galindo-Leal \& Camara, 2003). Se define como un bosque subtropical semi- deciduo, con un estrato arbóreo superior de más de $30 \mathrm{~m}$ de altura. Se caracteriza por veranos calientes (superiores a $35^{\circ} \mathrm{C}$ ) e inviernos con heladas. La temperatura media anual es de 16 a $22{ }^{\circ} \mathrm{C}$. Las precipitaciones varían entre los 1700 y $2200 \mathrm{~mm}$ anuales sin estaciones de sequías marcadas (Di Bitetti \& al., 2003).

Desde el punto de vista etnográfico, la región se caracteriza por su pluri-culturalidad (Zamudio \& al., 2010). En ésta conviven pueblos originarios Mby'a-guaraní, criollos e inmigrantes. Se definen como criollos a los pobladores de diferente origen cultural, que conviven en la zona, presentan una idiosincrasia regional (costumbres alimentarias, formas de uso de los recursos, sistemas productivos) compartida

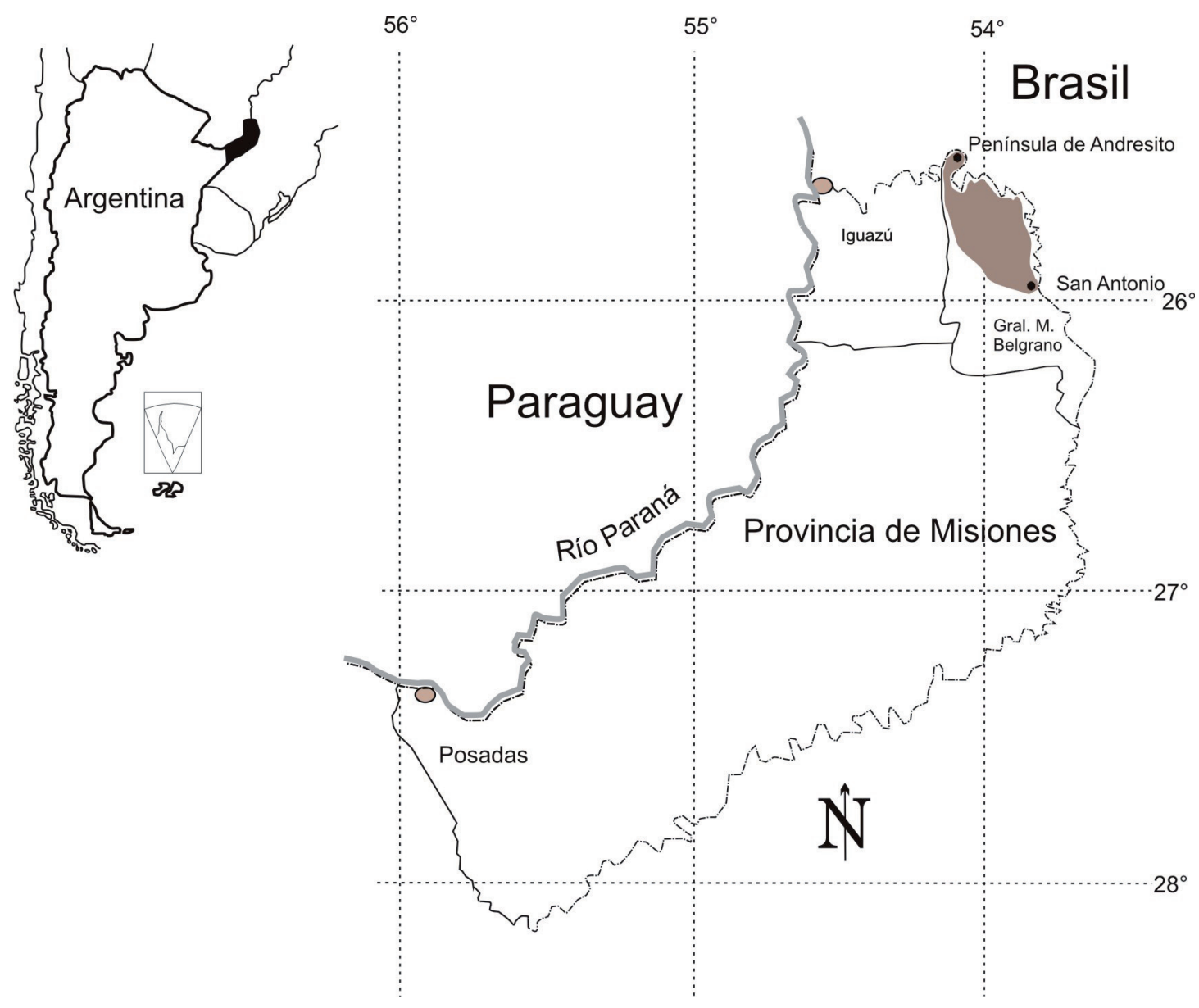

Fig. 1. Área de estudio. 
parcialmente con los vecinos brasileños. Hablan alternativamente portugués, español o "portuñol" (dialecto fruto de la combinación de ambos). En general, mantienen pocos vínculos con sus vecinos Mby'a-guaraníes. No obstante, también han incorporado vocablos de su idioma y/o de otras lenguas de la rama lingüística Tupí-guaraní.

Dentro del Departamento Gral. Manuel Belgrano se trabajó en diferentes poblados rurales, los que, en general, cuentan con infraestructura y servicios públicos mínimos. Dada la heterogeneidad cultural, se exploró una amplia región (5 caseríos o "picadas" distantes entre sí), de modo de captar la variabilidad local acerca de los conocimientos y percepciones sobre los recursos empleados. Esta región se puede definir en un radio que va desde el poblado de Península de Andresito (del municipio del mismo nombre) en el límite oeste del Parque Nacional Iguazú, hasta el paraje Alegría, en el Municipio de San Antonio. En su mayoría son pequeños productores $(5$ a $50 \mathrm{ha}$ ), propietarios o no. Obtienen ingresos monetarios a partir del cultivo de tabaco, o una combinación de éste con otras especies o actividades (por ejemplo, tabaco y yerba mate, o tabaco y trabajos rurales como jornaleros). La economía familiar se completa con cultivos destinados al autoconsumo (en particular, maíz, mandioca, zapallo y porotos) y la cría de animales (vacas, cerdos y aves de corral).

\section{Diseño metodológico}

El muestreo se realizó entre 2007 y 2009. La selección de informantes combinó el muestreo al azar con la técnica de bola de nieve, quedando así incluidos expertos y novicios (conocen menos y más de 9 etnoespecies de insectos productores de miel, respectivamente). Se trabajó con un total de 68 pobladores, 12 mujeres entre 24 y 66 años (promedio de 45) y 56 hombres entre 19 y 76 años (promedio de 51). Entre éstos, 36 son argentinos, 25 brasileños y 7 paraguayos. Cada informante fue visitado entre dos y seis veces.

Se efectuaron entrevistas abiertas y semiestructuradas (Bernard, 2000). Además, junto con los pobladores se recorrieron paisajes agrícolas, áreas de bosque y caminos rurales de modo de ubicar, reconocer y colectar muestras de abejas y plantas mencionadas. Las muestras de abejas se encuentran transitoriamente en la colección entomológica del Instituto de Biología Subtropical, Misiones (IBS-UNAM) y serán depositadas en el Museo Argentino de Ciencias Naturales "Bernardino Rivadavia" (MACN), Capital Federal. La mayor parte de las abejas sin aguijón colectadas fueron identificadas por especialistas (F. A. Silveira, C. Rasmussen); el resto, fue identificado hasta el nivel genérico por Fernando Zamudio (FZ). Las muestras de plantas fueron identificadas por Monika Kujawska (MK), Pablo Stampella (PS) y Norma Hilgert. La colección de MK está depositada en el Herbario CTES, del Instituto de Botánica del Nordeste, CONICET, los cítricos (PS) en el herbario del Laboratorio de Etnobotánica y Botánica Aplicada (LEBA), de la Facultad de Ciencias Naturales y Museo, UNLP y el resto (FZ) en la colección del Intituto de Biología Subtropical, UNAM.

\section{Análisis de datos}

Se cuantificó el número de usos medicinales de los productos obtenidos de las colonias de las abejas sin aguijón y de Apis mellifera según la especie de procedencia y su frecuencia de uso. Un reporte de uso fue considerado cuando algún producto, o combinación de productos, fue indicado para el tratamiento de una dolencia específica de acuerdo a la terminología local. Las dolencias reportadas fueron categorizadas por los autores según los sistemas corporales tratados, las formas de preparación y administración y el número de informantes que los citaron. Por otra parte, se consideran preparados medicinales compuestos a aquellos que incluyen la combinación de al menos dos sustancias (una de las cuales es la miel). Para analizar si hay relación entre la especie que produce la miel y el uso medicinal citado se realizó un Análisis de Componentes Principales. Para comparar el uso de las mieles más frecuentemente citadas según las dolencias tratadas se realizó un test de chi cuadrado (Di Rienzo \& al., 2009). 


\section{Resultados}

\section{Usos medicinales de los himenópteros melíferos regionales y sus productos}

Se reportaron un total de 412 usos correspondientes a 9 taxones. La miel es el principal producto utilizado como remedio $\mathrm{y}$ alimento funcional, considerándolo como tal cuando se consume como energizante, tonificante (por ejemplo, mejorar la piel y el cabello) o bueno para la salud. Además, en menor proporción, se usan diferentes partes del nido (piqueras, cera, cerumen y resinas) y el veneno de Apis mellifera. Las especies más citadas fueron Tetragonisca fiebrigi y A. mellifera (Tabla 1).

Tabla 1. Usos medicinales de las etnoespecies de abejas de la región y sus partes usadas.

\begin{tabular}{|c|c|c|c|c|c|c|c|}
\hline \multirow{2}{*}{ Etnoespecies } & \multirow{2}{*}{ Nombre Científico } & \multicolumn{5}{|c|}{ Usos } & \multirow{2}{*}{$\begin{array}{l}\text { Partes } \\
\text { Usadas }\end{array}$} \\
\hline & & Total & Med & $U t$ & Al Fun & Otros & \\
\hline Yateí & Tetragonisca fiebrigi & 222 & 214 & 4 & 2 & \multirow[t]{10}{*}{2} & $\mathrm{C}, \mathrm{M}$ \\
\hline Abeja & Apis mellifera & 148 & 141 & 2 & 5 & & $\begin{array}{l}\mathrm{C}, \mathrm{M} \\
\mathrm{A}, \mathrm{R}\end{array}$ \\
\hline Carabozá & Trigona spinipes & 12 & 12 & & & & M \\
\hline Borá & Tetragona clavipes & 10 & 10 & & & & M \\
\hline Iratín & Lestrimelitta limao o L. rufa & 7 & 5 & 2 & & & $\mathrm{P}$ \\
\hline Mandasaia & Melipona quadrifasciata & 5 & 4 & 1 & & & $\mathrm{M}, \mathrm{R}$ \\
\hline Miri & Plebeia spp. & 5 & 5 & & & & M \\
\hline Guaraipo & Melipona bicolor & 1 & 1 & & & & M \\
\hline Mambuca & Cephalotrigona capitata & 1 & 1 & & & & $\mathrm{R}$ \\
\hline Otras & varias especies de meliponinas & 2 & & 2 & & & $\mathrm{R}$ \\
\hline & TOTAL & 412 & 392 & 11 & 7 & 2 & \\
\hline
\end{tabular}

Med $=$ medicinales, $\mathrm{Ut}=$ utilitarios, $\mathrm{Al}$ Fun $=$ alimento funcional, $\mathrm{C}=$ cerumen o cera, $\mathrm{M}=$ miel, $\mathrm{P}=$ piquera (estructura de entrada a la colonia, generalmente tubular, construida con cera y resinas vegetales), $\mathrm{R}=$ resina $\mathrm{O}$ propóleo, $\mathrm{A}=$ aguijón.

Las mieles se usan para tratar dolencias de 9 sistemas corporales, siendo el respiratorio el que recibió un mayor número de reportes de uso $(84,3 \%)$, seguido de las dolencias de los sistemas dérmico y ocular, con una menor frecuencia marcada $(8,9$ y $2,6 \%$ respectivamente) (Tabla 2). Por otra parte la miel se emplea también como alimento funcional. Esta categoría de uso recibió 24 menciones ocupando un lugar intermedio entre los usos del sistema dérmico y ocular (segundo y tercer lugar de importancia).

La miel de T. fiebrigi fue mencionada exclusivamente para tratar diversas afecciones de los sistemas ocular, circulatorio y reproductivo. Por otra parte, la miel de $A$. mellifera recibió un uso exclusivo para los sistemas óseo-muscular y psicológico, y fue mencionada en una mayor frecuencia que la miel de T. fiebrigi como un alimento funcional y para tratar problemas digestivos.
Se reportaron frecuencias de usos similares de las mieles de T. fiebrigi y A. mellifera para tratar dolencias de los sistemas respiratorio y dérmico (Fig. 2 y Tabla 1).

Al relacionar los sistemas corporales tratados según las etnoespecies a través de un Análisis de Componentes Principales, se encontró que los ejes 1 y 2 explican el $88 \%$ de la variación de los datos. Se observa una agrupación (área A) que refleja la relación entre los productos obtenidos de T. fiebrigi y A. mellifera con las dolencias de los sistemas respiratorio, dérmico y digestivo. Así mismo, se visualiza el rol de la miel de $A$. mellifera como alimento funcional (área B); el de la miel de T. fiebrigi en el tratamiento de afecciones oculares (área C); y finalmente, con menos claridad, se esboza la relación entre el tratamiento de dolencias del sistema óseo-muscular y las mieles de "carabozá" (Trigona spinipes), "mirí" 
Tabla 2. Sistemas corporales, afecciones y formas de preparación de las medicinas domésticas.

\begin{tabular}{|c|c|c|c|c|}
\hline $\begin{array}{c}\text { Sistema } \\
\text { Corporal }\end{array}$ & \# citas & $\%$ & Afecciones & $\begin{array}{c}\text { Forma de } \\
\text { preparación }\end{array}$ \\
\hline Respiratorio & 321 & 84,3 & $\begin{array}{l}\text { Gripe, bronquitis, pulmonías, afecciones pulmonares, } \\
\text { tos, asma, resfrío, angina, catarro, afecciones de vías } \\
\text { respiratorias, dolor de garganta, tuberculosis, sinusitis }\end{array}$ & $\begin{array}{l}\text { I, J, D, P, M, } \\
\text { MA, C, F/M, S }\end{array}$ \\
\hline Dérmico & 34 & 8,9 & $\begin{array}{l}\text { Forúnculos (llamados localmente nacidos), } \\
\text { lastimaduras, para sacar espinas, quemaduras }\end{array}$ & $\mathrm{C}, \mathrm{P}$ \\
\hline Ocular & 10 & 2,6 & $\begin{array}{l}\text { Afecciones oculares (basura en los ojos, lastimaduras, } \\
\text { ojos cansados, ojos lagrimosos, cataratas). }\end{array}$ & $\mathrm{P}$ \\
\hline Óseo-muscular & 6 & 1,6 & Reuma, dolores musculares, calambres & $\mathrm{P}$ \\
\hline Digestivo & 5 & 1,3 & $\begin{array}{l}\text { Problemas digestivos (dolor de panza), constipación, } \\
\text { parasitosis* }\end{array}$ & $\mathrm{P}, \mathrm{M}, \mathrm{D}$ \\
\hline Psicológico & 2 & 0,5 & Estrés & $\mathrm{I}, \mathrm{P}$ \\
\hline Reproductivo & 1 & 0,3 & No especificado (“cuestiones de la mujer") & $\mathrm{s} / \mathrm{d}$ \\
\hline Nervioso & 1 & 0,3 & Dolor de cabeza & M \\
\hline Circulatorio & 1 & 0,3 & Diabetes & $\mathrm{D}$ \\
\hline
\end{tabular}

$\mathrm{I}=$ Infusión, $\mathrm{J}=$ jarabe, $\mathrm{D}=$ decocción, $\mathrm{P}=$ pura, $\mathrm{M}=$ mezcla, $\mathrm{MA}=$ macerado, $\mathrm{C}=$ cataplasma, $\mathrm{F} / \mathrm{M}=$ fritura/mezcla, $\mathrm{S}=$ sahumerio; * La miel se le aplica en la planta de los pies a los bebes o niños para que "las lombrices de la panza bajen en busca del la miel"

(Plebeia spp.) e "iratín" (Lestrimelitta limao y L. rufa) (Fig. 3). Al comparar los usos compartidos entre $T$. fiebrigi y $A$. mellifera identificados en la Tabla 2, no se hallaron diferencias significativas para las dolencias de los sistemas dérmico y digestivo; en cambio, sí las hay en las del sistema respiratorio $\left(\chi^{2}=15.01, \mathrm{P}=0.0001\right.$, $\alpha=0.05,1$ GL).

La miel se consume pura o preparada de 8 diferentes formas; entre éstas, las infusiones, jarabes y decocciones recibieron un mayor número de reportes (Tabla 3 y 4, Fig. 4). Al considerar las formas de preparación en relación a las dolencias, encontramos que la miel en decocciones, jarabes, infusiones, macerados, mezclas y fritura/mezclas, se utiliza principalmente para tratar dolencias del sistema respiratorio. Con excepción a la forma pura y las cataplasmas, las dolencias de este sistema superan el 50\% de las menciones. La miel pura se usa para tratar dolencias de los sistemas respiratorio $(47 \%)$, ocular $(10 \%)$ y óseo-muscular y dérmico (ambos con 5\%), en tanto que se ingiere como alimento funcional en el $10 \%$ de los casos. Las cataplasmas fueron mencionadas principalmente para tratar dolencias del sistema dérmico $(90 \%) \mathrm{y}$, en baja frecuencia, para dolencias del sistema respiratorio $(10 \%)$. En este caso, la miel se frota en un papel caliente y se pone sobre el pecho del enfermo o se unta sobre el pecho y se tapa con una tela. El uso en forma de sahumerio se mencionó en una sola ocasión para tratar sinusitis; el procedimiento consiste en quemar la piquera del nido de Lestrimelitta limao o L. rufa con una brasa, e inhalar el humo por la nariz, tapando la boca.

Según la frecuencia de citas, las mieles de $T$. fiebrigi y A. mellifera se preparan en proporciones semejantes cuando se trata de decocciones, infusiones, macerados y cataplasmas. En cambio, la miel de $T$. fiebrigi se usa más en mezclas en frío y pura, y la de A. mellifera en jarabes. El uso medicinal de la miel pura alcanza el $21,8 \%$ 


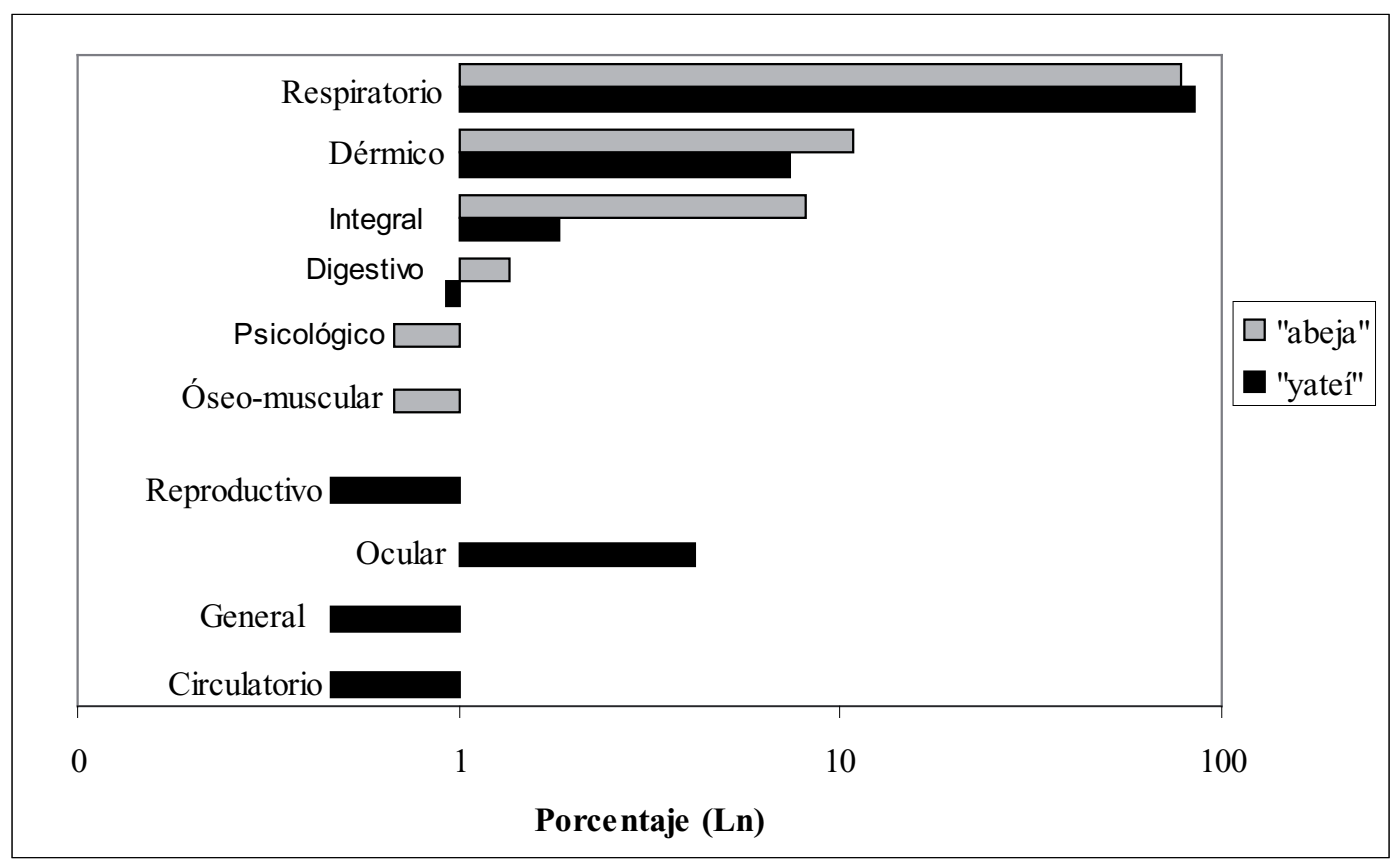

Fig. 2. Frecuencia de citas ( $\left.\mathrm{Ln}^{*}\right)$ de dolencias de los sistemas corporales tratados con la miel de Apis mellifera, "abeja”, y Tetragonisca fiebrigi, "yateí". * La transformación se realizó de modo de mejorar la visualización de las diferencias de los sistemas con menor número de menciones obliteradas por el elevado número de citas del sistema respiratorio.

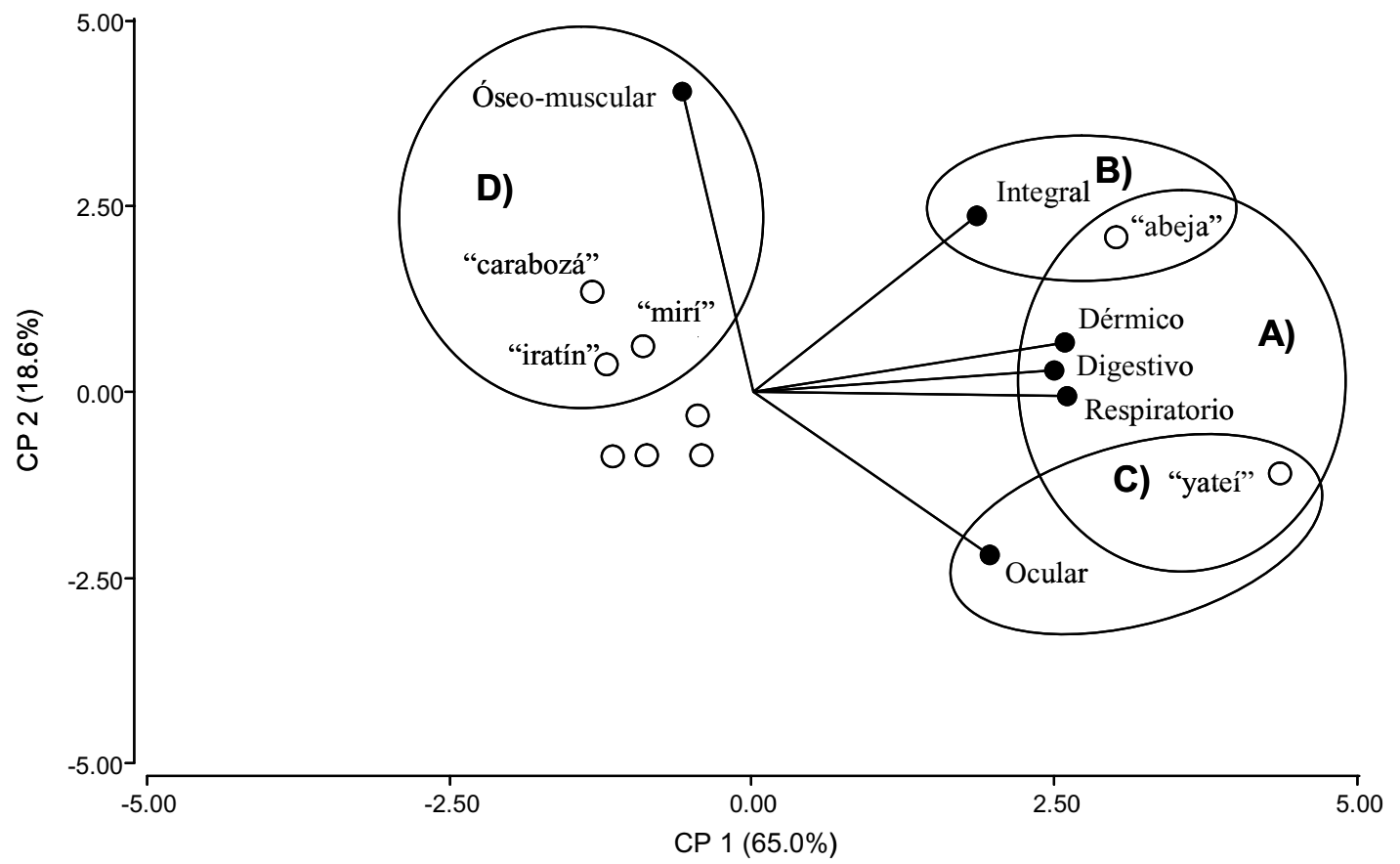

Fig. 3. Análisis de Componentes Principales donde se muestra las relaciones entre los sistemas corporales tratados y las etnoespecies. 


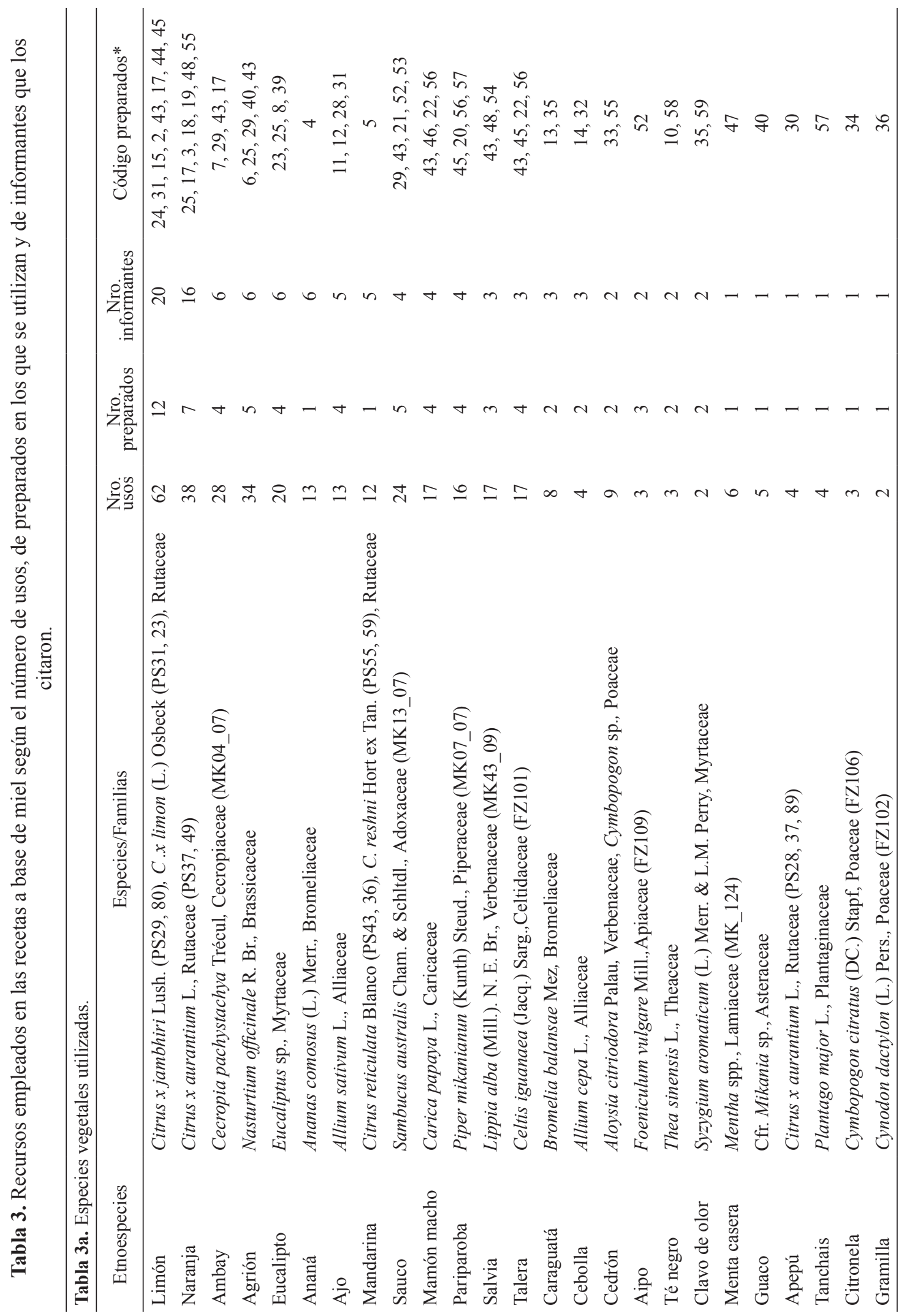


BONPLANDIA 20(2). 2011

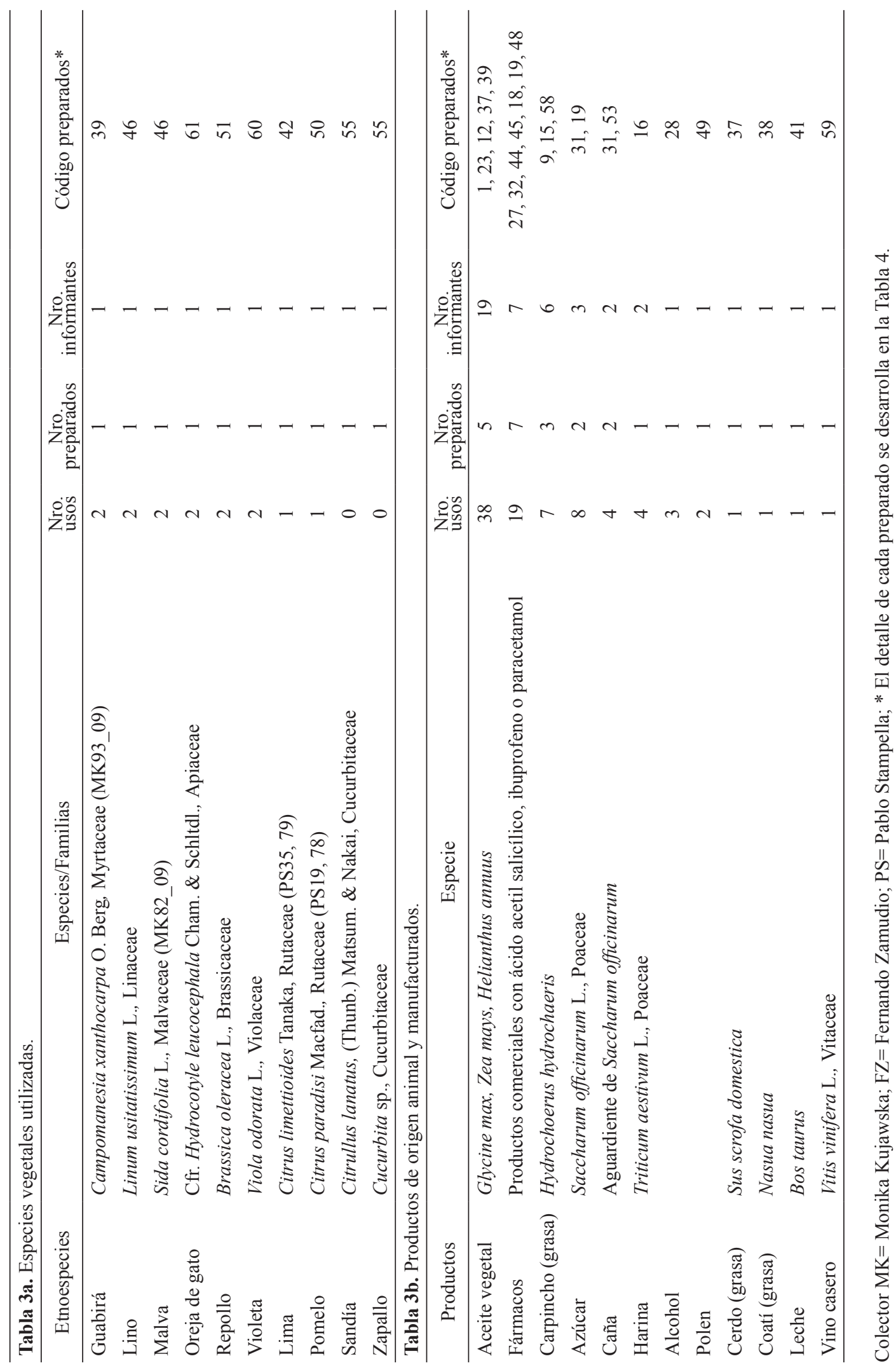




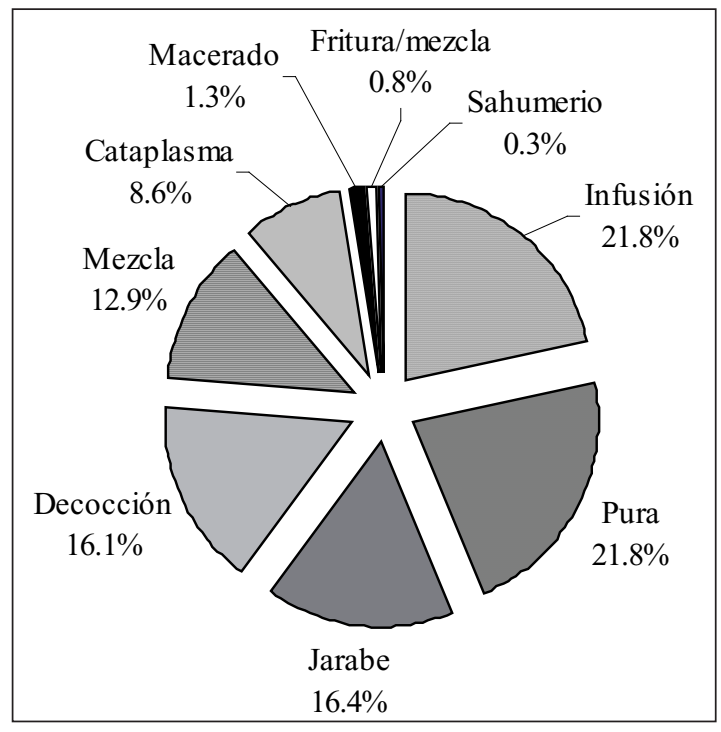

Fig. 4. Frecuencia de cita de cada forma de preparación (incluye todos los productos).

de las citas, el 78,2\% restante son mezclas con plantas, grasa o leche, fármacos y productos manufacturados como aceite o azúcar (Fig. 5). Dada la importancia de estos preparados, se expone a continuación un análisis sobre los componentes de las fórmulas y las combinaciones reportadas.

\section{Preparados medicinales a base de miel}

Los remedios caseros compuestos involucraron el uso de mieles de Tetragonisca fiebrigi (54\%), Apis mellifera (44\%) y Tetragona clavipes (2\%). Las mieles de $T$. fiebrigi y $A$. mellifera se utilizaron indistintamente en 33 preparados (54\%). El $31 \%$ de los preparados incluye exclusivamente miel de $T$. fiebrigi y el $15 \%$ de A. mellifera.

Se registraron 61 preparados mixtos en los que se combinan mieles (3 especies), plantas (35 etnoespecies, 39 especies), productos manufacturados (6) y de origen animal (5) (Tabla 3 y 4). Los preparados incluyen entre 1 y 7 elementos. El 64\% de los preparados fueron citados una vez, el $19,7 \%$ dos y el $16,4 \%$ restante entre 3 y 15 veces. Predominan los remedios con una especie de planta $(61 \%)$ y con un producto de origen animal o un fármaco $(36 \%)$.

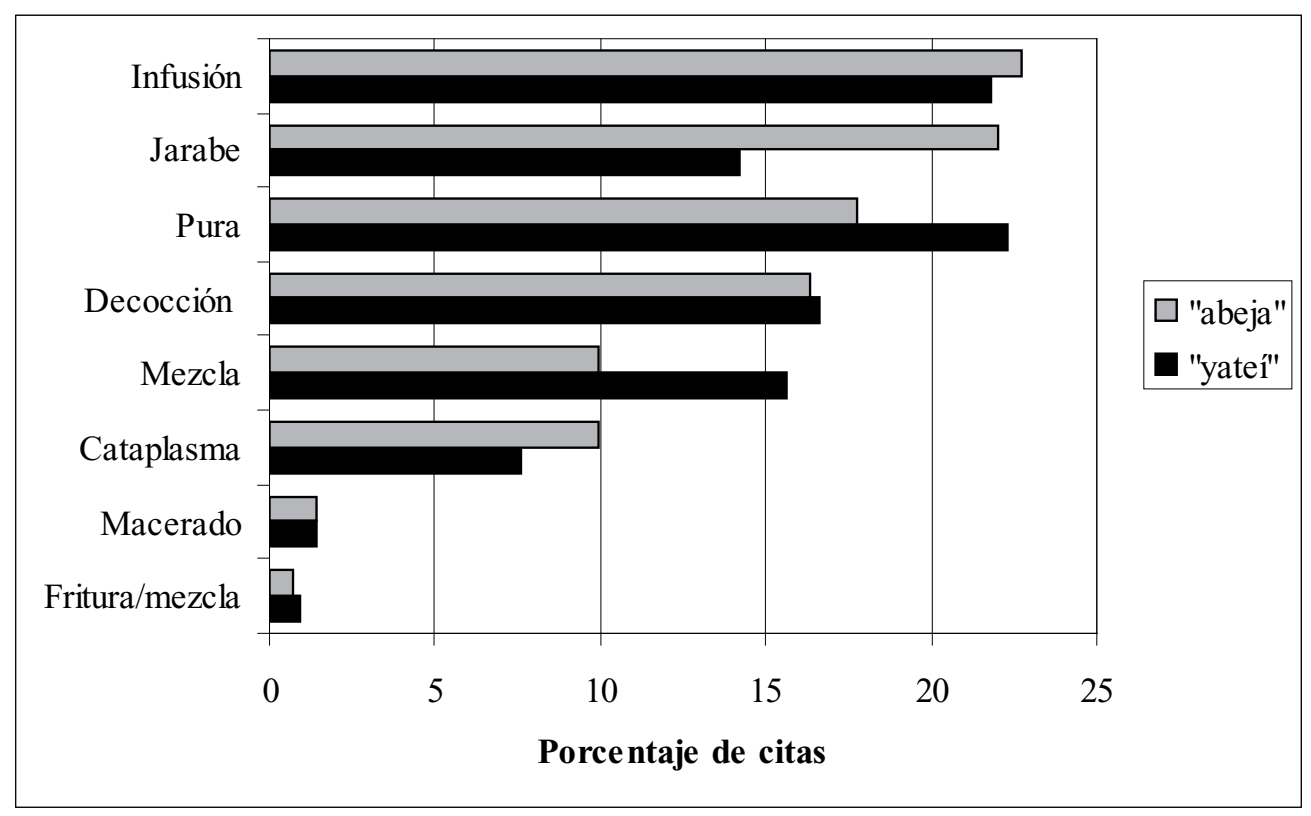

Fig. 5. Formas de preparación de las medicinas caseras a base de miel según las dos etnoespecies con mayor número de citas de uso. 
Tabla 4. Preparados medicinales realizados a base de miel.

\begin{tabular}{|c|c|c|c|c|c|c|}
\hline $\begin{array}{c}\text { Código } \\
\text { preparados }\end{array}$ & $\begin{array}{c}\text { Elemento/s } \\
\text { combinado/s con } \\
\text { miel }\end{array}$ & Dolencias & $\begin{array}{l}\text { Sistemas } \\
\text { Corporales }\end{array}$ & Preparación & $\begin{array}{l}\text { Adminis- } \\
\text { tración }\end{array}$ & $\begin{array}{c}\text { Nro. } \\
\text { informantes }\end{array}$ \\
\hline 1 & Aceite & $\begin{array}{l}\text { Asma, bronquitis y pulmonía, } \\
\text { catarro, gripe, constipación, } \\
\text { resfrío, tos }\end{array}$ & RE, DI & $\mathrm{M}$ & $\mathrm{O}$ & 15 \\
\hline 2 & Limón & $\begin{array}{l}\text { Angina, asma, bronquitis y } \\
\text { pulmonía, dolor de garganta, } \\
\text { gripe, resfrío, tos }\end{array}$ & $\mathrm{RE}$ & $\mathrm{I}, \mathrm{J}, \mathrm{M}$ & $\mathrm{O}$ & 9 \\
\hline 3 & Naranja & $\begin{array}{l}\text { Gripe, angina, bronquitis y } \\
\text { pulmonía, tos, forúnculos, estrés }\end{array}$ & $\begin{array}{l}\text { RE, DE, } \\
\quad \text { PS }\end{array}$ & CA, I & $\mathrm{T}, \mathrm{O}$ & 7 \\
\hline 4 & Ananá & $\begin{array}{l}\text { Bronquitis y pulmonía, gripe, } \\
\text { resfrío, tos, asma, angina }\end{array}$ & $\mathrm{RE}$ & $\mathrm{D}, \mathrm{J}$ & $\mathrm{O}$ & 6 \\
\hline 5 & Mandarina & Gripe, resfrío, tos, integral, nacido & RE, DE & I & $\mathrm{O}$ & 5 \\
\hline 6 & Agrión & $\begin{array}{l}\text { Bronquitis y pulmonía, dolor de } \\
\text { garganta, tos, gripe, resfrío }\end{array}$ & $\mathrm{RE}$ & $\mathrm{D}, \mathrm{J}$ & $\mathrm{O}$ & 4 \\
\hline 7 & Ambay & Bronquitis y pulmonía, gripe, tos & RE & $\mathrm{D}, \mathrm{J}$ & $\mathrm{O}$ & 4 \\
\hline 8 & Eucalipto & $\begin{array}{l}\text { Angina, bronquitis y pulmonía, } \\
\text { catarro, gripe, tos }\end{array}$ & $\mathrm{RE}$ & I, D & $\mathrm{O}$ & 3 \\
\hline 9 & $\begin{array}{l}\text { Grasa de } \\
\text { carpincho }\end{array}$ & Bronquitis y pulmonía & $\mathrm{RE}$ & M & $\mathrm{O}$ & 3 \\
\hline 10 & Té negro común & Bronquitis y pulmonía, tos & $\mathrm{RE}$ & I & $\mathrm{O}$ & 3 \\
\hline 11 & Ajo & Bronquitis y pulmonía, tos & $\mathrm{RE}$ & $\mathrm{D}$ & $\mathrm{O}$ & 2 \\
\hline 12 & Ajo, aceite & Tos & $\mathrm{RE}$ & $\mathrm{F} / \mathrm{M}$ & $\mathrm{O}$ & 2 \\
\hline 13 & Caraguatá & $\begin{array}{l}\text { Bronquitis y pulmonía, gripe, } \\
\text { integral }\end{array}$ & $\mathrm{RE}$ & $\mathrm{D}, \mathrm{J}$ & $\mathrm{O}$ & 2 \\
\hline 14 & Cebolla & Bronquitis y pulmonía & RE & $\mathrm{D}$ & $\mathrm{O}$ & 2 \\
\hline 15 & $\begin{array}{l}\text { Grasa de } \\
\text { carpincho, } \\
\text { limón }\end{array}$ & Bronquitis y pulmonía, gripe & $\mathrm{RE}$ & M & $\mathrm{O}$ & 2 \\
\hline 16 & Harina & Forúnculos & $\mathrm{DE}$ & $\mathrm{CA}$ & $\mathrm{T}$ & 2 \\
\hline 17 & $\begin{array}{l}\text { Limón, naranja, } \\
\text { ambay }\end{array}$ & Tos & $\mathrm{RE}$ & $\mathrm{D}, \mathrm{J}$ & $\mathrm{O}$ & 2 \\
\hline 18 & $\begin{array}{l}\text { Naranja, } \\
\text { fármaco }\end{array}$ & Dolor de garganta, gripe, tos & $\mathrm{RE}$ & I & $\mathrm{O}$ & 2 \\
\hline 19 & $\begin{array}{l}\text { Naranja, cedrón, } \\
\text { fármaco, azúcar }\end{array}$ & Gripe, tos & $\mathrm{RE}$ & I & $\mathrm{O}$ & 2 \\
\hline 20 & Pariparoba & $\begin{array}{l}\text { Lastimaduras, sacar espinas, } \\
\text { infecciones (por ejemplo, ura }{ }^{1} \text { mal } \\
\text { curada) }\end{array}$ & $\mathrm{DE}$ & $\mathrm{CA}$ & $\mathrm{T}$ & 2 \\
\hline 21 & Sauco & $\begin{array}{l}\text { Asma, resfrío, bronquitis y } \\
\text { pulmonía, gripe }\end{array}$ & $\mathrm{RE}$ & $\mathrm{D}, \mathrm{J}$ & $\mathrm{O}$ & 2 \\
\hline
\end{tabular}

${ }^{1}$ Parasitosis originada por las larvas de la mosca Dermatobia hominis que se desarrollan en el tejido subcutáneo. 
F. Zamudio \& N. I. Hilgert, Mieles y plantas en la medicina criolla

\begin{tabular}{|c|c|c|c|c|c|c|}
\hline $\begin{array}{l}\text { Código } \\
\text { preparados }\end{array}$ & $\begin{array}{c}\text { Elemento/s } \\
\text { combinado/s con } \\
\text { miel }\end{array}$ & Dolencias & $\begin{array}{c}\text { Sistemas } \\
\text { Corporales }\end{array}$ & Preparación & $\begin{array}{c}\text { Adminis- } \\
\text { tración }\end{array}$ & $\begin{array}{c}\text { Nro. } \\
\text { informantes }\end{array}$ \\
\hline 22 & $\begin{array}{l}\text { Talera, limón, } \\
\text { mamón macho }\end{array}$ & Gripe & RE & $\mathrm{D}, \mathrm{J}$ & $\mathrm{O}$ & 2 \\
\hline 23 & $\begin{array}{l}\text { Aceite, } \\
\text { eucalipto }\end{array}$ & Angina, bronquitis y pulmonía, tos & RE & I & $\mathrm{O}$ & 1 \\
\hline 24 & Aceite, limón & Bronquitis y pulmonía & RE & M & $\mathrm{O}$ & 1 \\
\hline 25 & $\begin{array}{l}\text { Agrión, naranja, } \\
\text { eucalipto }\end{array}$ & $\begin{array}{l}\text { Asma, bronquitis y pulmonía, } \\
\text { gripe }\end{array}$ & RE & $\mathrm{D}$ & $\mathrm{O}$ & 1 \\
\hline 26 & Aipo & Resfrío & RE & I & $\mathrm{O}$ & 1 \\
\hline 27 & Aipo, fármaco & Resfrío & RE & $\mathrm{D}$ & $\mathrm{O}$ & 1 \\
\hline 28 & Alcohol, ajo & Angina, bronquitis y pulmonía, tos & RE & MA & $\mathrm{O}$ & 1 \\
\hline 29 & $\begin{array}{l}\text { Ambay, sauco, } \\
\text { agrión }\end{array}$ & $\begin{array}{l}\text { Angina, bronquitis y pulmonía, } \\
\text { catarro, gripe, tos }\end{array}$ & RE & $\mathrm{D}$ & $\mathrm{O}$ & 1 \\
\hline 30 & Apepú & Bronquitis y pulmonía, gripe & RE & $\mathrm{J}$ & $\mathrm{O}$ & 1 \\
\hline 31 & $\begin{array}{l}\text { Aguardiente } \\
\text { de caña, limón, } \\
\text { ajo, azúcar }\end{array}$ & Bronquitis y pulmonía, gripe & RE & $\mathrm{D}$ & $\mathrm{O}$ & 1 \\
\hline 32 & $\begin{array}{l}\text { Cebolla, } \\
\text { fármaco }\end{array}$ & Tos & $\mathrm{RE}$ & $\mathrm{J}$ & $\mathrm{O}$ & 1 \\
\hline 33 & Cedrón & Bronquitis y pulmonía, gripe & RE & I & $\mathrm{O}$ & 1 \\
\hline 34 & Citronela & Gripe, resfrío, tos & $\mathrm{RE}$ & $\mathrm{J}$ & $\mathrm{O}$ & 1 \\
\hline 35 & $\begin{array}{l}\text { Clavo de olor, } \\
\text { caraguatá }\end{array}$ & Cuestiones de mujeres & $\mathrm{RP}$ & & & 1 \\
\hline 36 & Gramilla & Gripe & $\mathrm{RE}$ & I & $\mathrm{O}$ & 1 \\
\hline 37 & $\begin{array}{l}\text { Grasa de } \\
\text { chancho }\end{array}$ & Gripe & $\mathrm{RE}$ & M & $\mathrm{O}$ & 1 \\
\hline 38 & Grasa de coatí & Asma & RE & M & $\mathrm{O}$ & 1 \\
\hline 39 & $\begin{array}{l}\text { Guabirá, ajo, } \\
\text { eucalipto, } \\
\text { Aceite }\end{array}$ & Bronquitis y pulmonía & $\mathrm{RE}$ & $\mathrm{D}$ & $\mathrm{O}$ & 1 \\
\hline 40 & Guaco, agrión & $\begin{array}{l}\text { Asma, bronquitis y pulmonía, } \\
\text { gripe, tos, tuberculosis }\end{array}$ & RE & $\mathrm{J}$ & $\mathrm{O}$ & 1 \\
\hline 41 & Leche & Catarro & $\mathrm{RE}$ & M & $\mathrm{O}$ & 1 \\
\hline 42 & Lima & Tos & $\mathrm{RE}$ & I & $\mathrm{O}$ & 1 \\
\hline 43 & $\begin{array}{l}\text { Limón, ambay, } \\
\text { mamón macho, } \\
\text { agrión, sauco, } \\
\text { talera, salvia }\end{array}$ & $\begin{array}{l}\text { Angina, bronquitis y pulmonía, } \\
\text { catarro, gripe, tos }\end{array}$ & RE & $\mathrm{D}$ & $\mathrm{O}$ & 1 \\
\hline 44 & Limón, fármaco & Angina, gripe & $\mathrm{RE}$ & I & $\mathrm{O}$ & 1 \\
\hline 45 & $\begin{array}{l}\text { Limón, talera, } \\
\text { pariparoba }\end{array}$ & $\begin{array}{l}\text { Dolor de garganta, vías } \\
\text { respiratorias }\end{array}$ & RE & $\mathrm{D}$ & $\mathrm{O}$ & 1 \\
\hline
\end{tabular}




\begin{tabular}{|c|c|c|c|c|c|c|}
\hline $\begin{array}{c}\text { Código } \\
\text { preparados }\end{array}$ & $\begin{array}{c}\text { Elemento/s } \\
\text { combinado/s con } \\
\text { miel }\end{array}$ & Dolencias & $\begin{array}{c}\text { Sistemas } \\
\text { Corporales }\end{array}$ & Preparación & $\begin{array}{l}\text { Adminis- } \\
\text { tración }\end{array}$ & $\begin{array}{c}\text { Nro. } \\
\text { informantes }\end{array}$ \\
\hline 46 & $\begin{array}{l}\text { Malva, lino, } \\
\text { mamón macho }\end{array}$ & Gripe, tos & $\mathrm{RE}$ & I & $\mathrm{O}$ & 1 \\
\hline 47 & Menta casera & Gripe, resfrío, tos & RE & I & $\mathrm{O}$ & 1 \\
\hline 48 & $\begin{array}{l}\text { Naranja, salvia, } \\
\text { fármaco }\end{array}$ & Gripe & $\mathrm{RE}$ & $\mathrm{D}$ & $\mathrm{O}$ & 1 \\
\hline 49 & Polen & Tos & $\mathrm{RE}$ & M & $\mathrm{O}$ & 1 \\
\hline 50 & Pomelo & Gripe & $\mathrm{RE}$ & $\mathrm{D}$ & $\mathrm{O}$ & 1 \\
\hline 51 & Repollo & Forúnculos & $\mathrm{DE}$ & $\mathrm{CA}$ & $\mathrm{T}$ & 1 \\
\hline 52 & Sauco, aipo & Gripe & $\mathrm{RE}$ & $\mathrm{D}$ & $\mathrm{O}$ & 1 \\
\hline 53 & $\begin{array}{l}\text { Sauco, } \\
\text { aguardiente de } \\
\text { caña }\end{array}$ & Asma, resfrío & $\mathrm{RE}$ & MA & $\mathrm{O}$ & 1 \\
\hline 54 & Salvia & Gripe, resfrío, tos & $\mathrm{RE}$ & I & $\mathrm{O}$ & 1 \\
\hline 55 & $\begin{array}{l}\text { Sandía, zapallo, } \\
\text { naranja, } \\
\text { fármaco }\end{array}$ & No especificado & $\mathrm{RE}$ & $\mathrm{n} / \mathrm{d}$ & & 1 \\
\hline 56 & $\begin{array}{l}\text { Talera, limón, } \\
\text { mamón macho, } \\
\text { pariparoba }\end{array}$ & Dolor de garganta & $\mathrm{RE}$ & $\mathrm{J}$ & $\mathrm{O}$ & 1 \\
\hline 57 & $\begin{array}{l}\text { Tanchais, } \\
\text { pariparoba, } \\
\text { limón }\end{array}$ & Bronquitis y pulmonía, gripe & $\mathrm{RE}$ & $\mathrm{D}, \mathrm{I}$ & $\mathrm{O}$ & 1 \\
\hline 58 & $\begin{array}{l}\text { Té negro } \\
\text { común, grasa de } \\
\text { carpincho }\end{array}$ & Bronquitis y pulmonía & RE & M & $\mathrm{O}$ & 1 \\
\hline 59 & $\begin{array}{l}\text { Vino casero, } \\
\text { clavo de olor }\end{array}$ & Tos & $\mathrm{RE}$ & $\mathrm{D}$ & $\mathrm{O}$ & 1 \\
\hline 60 & Violeta & Forúnculos & $\mathrm{DE}$ & $\mathrm{CA}$ & $\mathrm{T}$ & 1 \\
\hline 61 & Oreja de gato & Forúnculos & $\mathrm{DE}$ & $\mathrm{CA}$ & $\mathrm{T}$ & 1 \\
\hline
\end{tabular}

$\mathrm{RE}=$ Respiratorio, $\mathrm{DE}=$ Dérmico, $\mathrm{DI}=$ Digestivo, $\mathrm{RP}=$ Reproductivo, $\mathrm{PS}=$ Psicológico, $\mathrm{O}=$ oral, $\mathrm{T}=$ tópico

Los vegetales más usados son Citrus $x$ jambhiri y C. $x$ limon (20 citas), Citrus $x$ aurantium (16), Cecropia pachystachya (6), Nasturtium officinale (6), Eucaliptus sp. (6) y Ananas comosus (6). Estas presentan diferentes grados de versatilidad de uso. Por ejemplo, C.x jambhiri y C.x limon se incluyen en 12 preparados diferentes, en tanto que $A$. comosus fue citado por seis informantes para realizar un mismo tipo de jarabe que consiste en la decocción lenta de miel junto con el fruto. Cecropia pachystachya fue la especie nativa con mayor acuerdo de uso, seguido por Sambucus australis, Piper mikanianun, Celtis iguanaea, Bromelia balansae y la adventicia Foeniculum vulgare. Entre éstas, S. australis fue la que presentó mayor versatilidad de uso: se emplea en cinco preparados diferentes, seguida de $C$. pachystachya, con cuatro citas (Tabla 3). 


\section{Discusión}

\section{Usos medicinales de los himenópteros melíferos regionales y sus productos}

El papel preponderante de la miel como alimento y medicina por sobre otros productos obtenidos observado en la región, concuerda parcialmente con estudios realizados con indígenas y criollos en América (Alves \& Rosa, 2006; Arenas, 2003; Cebolla, 2009; CostaNeto \& Oliveira, 2000; Ferreira \& al., 2009; Hill \& al., 1984; Modro \& al., 2009; Posey, 1983a, b). Sin embargo, entre los indígenas se reporta mayor diversidad de recursos usados, entre ellos larvas y polen como alimento y medicina, cerumen como pegamento y para la confección de artesanías y velas (Arenas, 2003; Cebolla, 2009; Falchetti \& Nates Parra, 2002; Hill \& al., 1984; Posey, 1983a, b).

El uso concomitante de especies de abejas sin aguijón con $A$ mellifera en comunidades de criollos, afro descendientes e indígenas, sugieren la coexistencia e influencia de tradiciones de uso (Alves \& Rosa, 2006; Arenas, 2003; Cebolla, 2009; Costa-Neto \& Oliveira, 2000; Ferreira \& al., 2009; Hill \& al., 1984; Martínez, 2008; Modro \& al., 2009; Posey, 1983b). En este sentido, se ha registrado entre los Ache de Paraguay que las mieles y larvas obtenidas de $A$ mellifera son más importantes en la dieta, dado que aportan más calorías que la suma del resto de mieles consumidas (Hill \& al., 1984). En cambio, en el contexto ceremonial los Mby'a de Misiones, usan la miel de "jate'i" o "mandori" ("yateí": T. fiebrigi; "manduri": Melipona obscurior, para los criollos) por ser consideradas "mieles genuinas" y, por el contrario, no se emplea para este fin la miel de $A$. mellifera porque se asume que es una creación de los blancos y no de Ñande Ru Pa (Cebolla, 2009).

Los pobladores estudiados reconocen un total de 12 etnoespecies de abejas sin aguijón (Zamudio \& Hilgert, 2012); para 9 de ellas se reportaron usos medicinales, entre las cuales la miel de T. fiebrigi, junto con la de A. mellifera, se destaca tanto en número de menciones como en diversidad de preparados. En un estudio reciente se ha hallado que estas dos especies son además las de mayor importancia relativa, tanto para los criollos como colonos de origen polaco que habitan el norte de Misiones (Kujawska \& al., 2011). En la misma provincia, los Mby'a-guaraní consideran a "jate'i" (T. angustula) como las más valorada de las meliponinas, por ser su miel la preferida en rituales como por sus propiedades medicinales (Cebolla, 2009).

De la literatura se deduce que la importancia relativa de los usos medicinales de las mieles varía según las especies y las comunidades estudiadas (Alves, 2009; Alves \& Rosa, 2007; Costa-Neto, 2002; Ferreira \& al., 2009). Si bien en el presente no se analizan los factores que podrían definir estas preferencias, en el futuro sería interesante explorar si las mismas están influenciadas por factores ecológicos, culturales y/o entomológicos.

Las mieles se usan de forma semejante a los reportes de la literatura donde se administra para tratar principalmente dolencias de los sistemas respiratorio, dérmico y digestivo (Alves, 2009; Alves \& Rosa, 2007; CostaNeto, 2002; Costa-Neto \& Oliveira, 2000; Ferreira \& al., 2009; Modro \& al., 2009). En la Argentina se reportan usos semejantes de mieles de diferentes especies para los mismos sistemas referidos, aunque son reportes aislados en trabajos de corte etnobotánico, a excepción del realizado por Cebolla (2009) que, al igual que nuestro trabajo, se centra en las "mieles de monte" (Arias Toledo \& al., 2007; Cebolla, 2009; Martínez, 2008; Martínez \& al., 2004; Scarpa, 2004).

Los resultados obtenidos sugieren la existencia de un patrón de uso general semejante de las mieles de $T$. fiebrigi y $A$. mellifera, dentro de un sistema donde se observan especificidades. La miel de ambas especies son las principalmente usadas para tratar dolencias del sistema respiratorio pero, inclusive dentro de este sistema, se usan de forma diferente. Es decir, ciertas dolencias son tratadas de forma específica con mieles de una u otra especie, por lo que pueden ser considerados recursos medicinales complementarios y $a$ priori no intercambiables.

Por otra parte, el uso de la miel de $A$. mellifera como alimento funcional es vox populi, en tanto que el uso oftalmológico de la miel de T. fiebrigi coincide con los reportes de 
uso de mieles de algunas especies de abejas sin aguijón en diferentes países de América, donde se aplica para tratar cataratas, glaucomas y pterigión (Costa-Neto, 2002; Costa-Neto \& Oliveira, 2000; Ferreira \& al., 2009). En Argentina tanto indígenas Mby'a como Tobas hacen un uso semejante de la miel de algunas meliponinas: Plebeia sp. (Martínez, 2008) y T. angustula (Cebolla, 2009; Martínez, 2008). El alto contenido de agua de las mieles de abejas Meliponini las vuelven más líquidas, lo cual posibilita el uso tópico en ojos y oídos (Ferreira \& al., 2009; Vit \& al., 2004). En México, un uso semejante se asignó a la miel de una avispa, Polybia occidentalis nigratella (Ramos-Elorduy \& Pino, 2003).

Por otra parte, el uso de la miel de Trigona spinipes y Plebeia spp., y el registro de Lestrimelitta limao y L. rufa para tratar dolencias del sistema óseo-muscular (reuma, dolores musculares y calambres), es exclusivo para estas etnoespecies en este estudio, y no ha sido reportada para otras Meliponini en la región. Para tratar la artrosis se ha documentado el uso de veneno de $A$. mellifera (Costa-Neto, 2002) en tanto que cataplasmas preparadas con agua de "contrayerba" (Trixis divaricata) y miel de A. mellifera, colocada sobre la zona afectada, es un remedio para golpes y dolores musculares entre los criollos del centro de la Argentina (Arias Toledo \& al., 2007).

Son escasos en la literatura los detalles sobre los modos de preparación y administración de la miel, a pesar de que se encuentra información valiosa sobre cómo un remedio casero puede ser eficiente terapéuticamente en términos del uso adecuado de los ingredientes, dosis y tiempo de preparación (Costa-Neto, 2004). Entre los criollos, se destacan los preparados sometidos a algún grado de procesamiento térmico, incluidas decocciones y jarabes que implican "hervir" o "cocinar la miel", lo cual podría incidir negativamente en las propiedades medicinales de la miel (Subramanian \& al., 2007). Esto podría ser particularmente perjudicial para la miel de A. mellifera de menor contendido de agua que las mieles de las abejas sin aguijón (Vit \& al., 2004). Dado que no se observó el proceso de preparación de los remedios caseros reportados, se debería profundizar sobre lo que implica "hervir" y "cocinar" la miel para los pobladores locales y realizar pruebas de laboratorio para evaluar los efectos de este procesamiento sobre él o los principios activos de las mieles.

Las diferencias observadas en los modos de preparación de las mieles de T. fiebrigi y $A$. mellifera están asociadas al tipo de "remedio" para el cual se utilizan. Por ejemplo, el uso frecuente de la miel de T. fiebrigi pura se relaciona a usos oftalmológicos, a pesar de que fue también la más utilizada en preparados compuestos, donde es usada principalmente en mezclas con aceite.

\section{Preparados medicinales a base de miel}

El uso de la miel en la preparación de remedios compuestos reportados en este trabajo, ha sido documentado en estudios etnozoológicos y etnobotánicos en América y el Caribe (Alves \& Rosa, 2007; Costa-Neto, 2002; Ferreira \& al., 2009; Hernández Cano \& Volpato, 2004; Schwarz, 1948; Modro \& al., 2009; Vandebroek \& al., 2010). Sin embargo, en las citas se suelen encontrar pocas especificaciones sobre identidad de los taxones involucrados en las mezclas ( $i c o n$ qué especies se mezclan?), las combinaciones (¿cómo se combinan?) y el consenso de uso dentro de la población estudiada. Esto ha sido señalado por Vandebroek \& al. (2010) como una tendencia de los estudios etnobiológicos de analizar los usos de los taxones de forma individual por sobre las formas en que se combinan los elementos que componen los preparados medicinales.

En cambio en los estudios realizados en Cuba (Hernández Cano \& Volpato, 2004) y en República Dominicana (Vandebroek \& al., 2010) se analizan las mezclas medicinales tradicionales, ampliamente difundidas en los países del Caribe. En general, estos preparados compuestos son más complejos en su elaboración y presentan un mayor número de componentes que los reportados en la población estudiada (Kujawska \& al, 2011; Zamudio \& al., 2010).

Las recetas descriptas en ambos estudios 
del Caribe y entre los pobladores del norte de Misiones se asemejan en dos aspectos; por un lado, son altamente variables en su conformación $\mathrm{y}$, por el otro, presentan un acuerdo de uso elevado según el tipo de dolencias tratadas. Entre los criollos, los preparados a base de miel se realizan principalmente para tratar dolencias del sistema respiratorio, en tanto que los reportados para los países del Caribe presentan un alto grado de correlación en la utilización de partes leñosas (cortezas) y raíces para el tratamiento de la salud reproductiva y condiciones genitourinarias, y de mezclas, o té, elaborados con partes frescas de plantas, para enfermedades respiratorias (Hernández Cano \& Volpato, 2004; Vandebroek \& al., 2010).

En cuanto a los patrones de uso y estructura de las combinaciones, la comparación con el estudio realizado por Hernández Cano \& Volpato (2004) en Cuba merece una discusión aparte, ya que este fue utilizado como modelo para el análisis de las mezclas entre los criollos. Por un lado, en ambos estudios la proporción entre el número de especies y el número de recetas (35/61 en Misiones) es menor a uno $(0,57$ en Misiones y 0,85 en Cuba) lo cual indica que las especies utilizadas se repiten muy pocas veces en los preparados. De allí que las recetas con una sola mención corresponden al $64 \%$ en este estudio y al $94 \%$ en Cuba. En tanto que las especies con mayor consenso de uso - el "limón" (Citrus x limon, Citrus $x$ jambhiri) entre los criollos, y el "coco" (Cocos nucifera) entre los cubanosse repiten en un 20 y un $15 \%$ de los preparados compuestos respectivamente. Es decir, existe consenso en el uso de algunos productos pero no en las combinaciones en la que éste y otros productos de menor consenso son mezclados.

En nuestro caso, el mayor consenso de uso se vincula a plantas y productos industrializados comúnmente usados en el tratamiento de dolencias respiratorias y fácilmente accesibles, por ser plantas cultivadas, o ruderales, y productos obtenidos en tiendas, o en la misma unidad productiva, ("limón", "ambay", "agrión", aceite vegetal). Según Vandebroek $\&$ al. (2010) el uso predominante de mezclas preparadas en té por no especialistas para tratar enfermedades respiratorias como la gripe, resfriado, tos, asma y bronquitis refleja el conocimiento general de las plantas cultivadas en el ámbito familiar y de las disponibles en los mercados, para el tratamiento de dolencias para las que a menudo no se busca atención médica especializada. En la zona de estudio la selección de los productos a incluir en un preparado medicinal está fuertemente moldeada por factores tales como disponibilidad, acceso y preferencias personales (Kujawska \& al., 2011).

De un total de 199 preparados reportados por Hernández Cano \& Volpato (2004), 13 usan miel (sin especificar el taxón) en combinación con un número variable de plantas. Diez de ellos se emplean para dolencias del sistema respiratorio (catarro, asma, neumonía y tuberculosis) de forma coincidente con este estudio. Al igual que lo observado aquí, en otros trabajos se reporta la combinación de la miel con aceite vegetal y grasa animal (Alves \& Rosa, 2007; Costa-Neto, 2004; Costa-Neto \& Motta, 2010; Hernández Cano $\&$ Volpato, 2004). Otro producto de origen animal también reportado en la literatura en combinación con miel es la leche de diversos animales domésticos (Ferreira $\&$ al., 2009). Entre los criollos de nuestro estudio la combinación de leche y miel se usa como alimento funcional, a diferencia de lo observado entre los descendientes de polacos en la misma zona, quienes lo consideran un recurso medicinal contra la tos y el dolor de garganta (Kujawska \& al., 2011).

En la región de estudio, el rol de la miel en los preparados y, en general, de todos sus componentes, es en la mayoría de los casos medicinal, con algunas excepciones donde es utilizada como saborizante (para que agarre el chupete). En la percepción local, todos los productos que forman parte de los preparados presentan propiedades que, en conjunto, "se ayudan" y/o complementan para dar más fuerza a la medicina (Kujaswka \& al., 2011). El aceite, y en especial la grasa de "carpincho" (Hydrochaeris hydrochaeris), actúa como descongestivo de mucosidades en tanto que para Hernández Cano \& Volpato (2004), el aceite y la grasa animal puede servir como un medio líquido que permite que los productos 
vegetales contenidos en las mezclas sean ingeridos con mayor facilidad.

\section{Conclusiones}

Dada la diversidad de himenópteros productores de miel presentes en América, en especial la relacionada a las abejas sin aguijón, y los patrones de usos reportados es adecuado considerar la identidad de los insectos que la produjeron. En la región estudiada se observó que las mieles de unas pocas especies conocidas son empleadas como medicinales, y que, dentro de éstas, las más importantes constituyen recursos medicinales complementarios. En el futuro, será interesante indagar cómo los factores como disponibilidad, acceso y preferencias personales, influyen frente a la ausencia de algunos de estos recursos.

El estudio de las combinaciones de productos de origen vegetal y animal, requiere de una aproximación interdisciplinaria más profunda, de modo de identificar qué "cortes" del corpus reflejan más adecuadamente la perspectiva emic y explican mejor los factores que modelan el uso de estos recursos.

\section{Agradecimientos}

A los pobladores rurales, por compartir sus conocimientos. A P. Tubaro, F. Silveira, C. Rasmussen, M. Kujawska y P. Stampella por su contribución en la identificación del material colectado. Al CONICET y la Fundación Rufford por el apoyo económico. A nuestros colegas del CeIBA e IBS por su apoyo permanente. A D. Varela y la Reserva Yateí, de Conservación Argentina, por el apoyo logístico y al proyecto en general.

\section{Bibliografía}

AHMAD, F., S. RAJ JOSHI \& M. B. GURUNG. 2003. The Himalayan cliff bee Apis laboriosa Smith and the honey hunters of Kaski. International Centre for Integrated Mountain Development, Kathmandu. 71 pp.

ALEXIADES, M. 2010. Apresentação. En U. P. Albuquerque, R. Farías Paiva de Lucena \& L. Vital
Fernandes Cruz da Cunha (eds.), Métodos e Técnicas na Pesquisa Etnobiológica e Etnoecológica, pp. 17. NUPEEA, Recife, Brazil.

ALVES, R. R. 2009. Fauna used in popular medicine in Northeast Brazil. J. Ethnobiol. Ethnomed. 5: 1.

\& I. L. ROSA. 2006. From cnidarians to mammals: The use of animals as remedies in fishing communities in NE Brazil. J. Ethnopharmacol. 107: 259-276.

- 2007. Zootherapeutic practices among fishing communities in North and Northeast Brazil: A comparison. J. Ethnopharmacol. 111: 82-103.

ARENAS, P. 2003. Etnografía y Alimentación entre los Tobañachilamole\#ek y Wichí-lhuku'tas del chaco central (Argentina). P. Arenas, Buenos Aires. 562 pp.

ARIAS TOLEDO, B., L. GALETTO \& S. COLANTONIO. 2007. Uso de plantas medicinales y alimenticias según características socioculturales en Villa Los Aromos (Córdoba, Argentina). Kurtziana 33: 79-88.

BANDEIRA, F. P. S. F. 2010. Prefácio. En U. P. Albuquerque, R. Farías Paiva de Lucena \& L. Vital Fernandes Cruz da Cunha (eds.), Métodos e Técnicas na Pesquisa Etnobiológica e Etnoecológica, pp. 11. NUPEEA, Recife, Brazil.

BERNARD, R. H. 2000. Social Research Methods. Qualitative and Quantitative Approaches. Sage Publications, London. 659 pp.

BUNGE, M. 1983. Epistemology \& Methodology II: Understanding the World. Reidel, Dordrecht. 296 pp.

CADOGAN, L. 1957. Arandu pova va'e Jakaira gui (Los que reciben buena ciencia de los Jakaira). Boletín de la Sociedad Científica del Paraguay. 2: 41-62.

CEBOLLA, M. V. 2009. El conocimiento y consumo de himenópteros, coleópteros y lepidópteros en la cultura Mbya-Guaraní, Misiones, Argentina. En E. M. Costa-Neto, D. Santos Fita \& M. V. Clavijo (eds.), Manual de Etnozoología, pp. 215-223. Tundra, Valencia.

COSTA-NETO, E. M. 1998. Folk Taxonomy and cultural significance of "Abeia" (Insecta: Hymenoptera) to the Pankararé, Northeastern Bahia State, Brazil. J. Ethnobiol. 18: 1-13.

- 2002. The Use of Insects in Folk Medicine in the State of Bahia, Northeastern Brazil, With Notes on Insects Reported Elsewhere in Brazilian Folk Medicine. Human Ecology 30: 245-263.

- 2004. Implications and applications of folk zootherapy in the state of Bahia, northeastern Brazil. Sustainable Development 12: 161-174.

— \& M. V. M. OLIVEIRA. 2000. Cockroach is good for asthma: zootherapeutic practices in Northeastern Brazil. Hum. Ecol. Rev. 7: 41-51.

\& P. C. MOTTA. 2010. Animal Species Traded 
as Ethnomedicinal Resources in the Federal District, Central West Region of Brazil. The Open Complementary Medicine Journal. Special Issue: Medical Ethnobiology, vol. 2, pp. 24-30.

DI BITETTI, M. S., G. PLACCI \& L. A. DIETZ. 2003. A Biodiversity Vision for the Upper Paraná Atlantic Forest Eco-region: Designing a Biodiversity Conservation Landscape and Setting Priorities for Conservation Action. WWF, Washington DC. 104 pp.

DI RIENZO, J. A., F. CASANOVES, M. G. BALZARINI, L. GONZALEZ, M. TABLADA \& C. W. ROBLEDO. 2009. InfoStat. Grupo InfoStat, FCA, Universidad Nacional de Córdoba.

ETKIN, N. L. 1994. Eating on the wild side. University of Arizona Press, Tucson. 305 pp.

— \& P. J. ROSS. 1982. Food as medicine and medicine as food: An adaptative framework for the interpretation of plant utilization among the Hausa of northern Nigeria. Soc. Sci. Med. 16: 1559-1573.

FALCHETTI, A.M. \& G. NATES PARRA. 2002. Las hijas del sol. Las abejas sin aguijón en el mundo uwa, Sierra Nevada del Cocuy, Colombia. En A. Ulloa (ed.), Rostros culturales de la fauna. Las relaciones entre los humanos y los animales en el contexto colombiano, pp. 175-214. Instituto Colombiano de Antropología e Historia, Bogotá.

FERREIRA, F. S., S. V. BRITO, S. C. RIBEIRO, W. O. ALMEIDA \& R. R. ALVES. 2009. Zootherapeutics utilized by residents of the community Poço Dantas, Crato-CE, Brazil. J. Ethnobiol. Ethnomed. 5: 21.

GALINDO-LEAL, C. \& I. G. CAMARA. 2003. Atlantic Forest Hotspot Status: An Overview. En C. GalindoLeal \& I. G. Camara (eds.), The Atlantic Forest of South América: biodiversity status, threats, and outlook, pp. 3-11. Island Press, Washington DC.

HANAZAKI, N., N. PERONI \& A. BEGOSSI. 2006. Edible and healing plants in the ethnobotany of native inhabitants of the Amazon and Atlantic Forest areas of Brazil. En A. Pieroni \& L. L. Price (eds.), Eating and Healing: Traditional Food as Medicine, pp. 251-271. Haworth Press, New York.

HERNÁNDEZ CANO, J. \& G. VOLPATO. 2004. Herbal mixtures in the traditional medicine of Eastern Cuba. J. Ethnopharmacol. 90: 293-316.

HILGERT, N. I. \& G. E. GIL. 2006. Plants of the Las Yungas Biosphere Reserve, Northwest of Argentina, used in health care. Biodiversity \& Conservation 15: 2565-2594.

HILL, K., K. HAWKES, M. HURTADO \& H. KAPLAN 1984. Seasonal Variance in the Diet of Ache HunterGatherers in Eastern Paraguay. Hum. Ecol. 12: 101-35.

KUJAWSKA, M. F. ZAMUDIO \& N. I. HILGERT. 2011. Honey-based mixtures used in home medicine by non-indigenous population of Misiones, Argentina. Evidence-Based Complementary and Alternative Medicine. ACEPTADO septiembre 2011 http:// www.hindawi.com/journals/ecam/aip/579350/.

LADIO A. H. 2010. Apresentação. En U. P. Albuquerque, R. Farías Paiva de Lucena \& L. Vital Fernandes Cruz da Cunha (eds.), Métodos e Técnicas na Pesquisa Etnobiológica e Etnoecológica, pp. 19. NUPEEA, Recife.

MAHAWAR M. M. \& D. P. JAROLI. 2007. Traditional knowledge on zootherapeutic uses by the Saharia tribe of Rajasthan. Indian J. Ethnobiol. Ethnomed. 25: 1-6.

MAHNER, M. \& M. BUNGE. 2000. Fundamentos de Biofilosofía. Siglo XXI, México, 462 pp.

MARTÍNEZ, G. J. 2008. La farmacopea natural en la etnomedicina de los tobas del Río Bermejito (Chaco, Argentina). Tesis doctoral, Facultad de Ciencias Agropecuarias, Universidad Nacional de Córdoba, Argentina. 289 pp.

MARTÍNEZ, M. R., M. L. POCHETTINO \& A. R. CORTELLA. 2004. Environment and illness in the Calchaquí Valley (Salta, Argentina): phytotherapy for osteo-articular and cardio-circulatory diseases. J. Ethnofarmacol. 95: 317-327.

MARTÍNEZ-CROVETTO, R. 1968. La alimentación entre los guaraníes de Misiones. Etnobiológica 4: $1-24$.

MEDA, A., C. LAMIEN, E. J. MILLOGO, M. ROMITO \& O. G. NACOULMA. 2004. Therapeutic uses of honey and honeybee larvae in central Burkina Faso. J. Ethnopharmacol. 95: 103-107.

MEDRANO, M. C. \& C. N. ROSSO. 2009. De la utilización de mieles nativas por Guaycurúes, una aproximación etnobiológica. Biológica 10: 38-43.

MODRO, A. F. H., S. SOUZA, F. H. ABURAYA \& E. MAIA. 2009. Conhecimento dos moradores do médio Araguaia, Estado do Mato Grosso, sobre a utilidade de produtos de abelhas (Hymenoptera, Apidae). Acta Sci. Biol. Sci. 31 (4): 421-424.

NGOKWEY, N. 1995. Home remedies and doctors' remedies in Feira (Brazil). Social Science \& Medicine 40: 1141-1153.

NOGUEIRA-NETO, P. 1997. Vida y criação de abelhas indígenas sem ferrão. Ed. Nogueirapis. São Paulo. Brasil. 446 pp.

PIERONI, A. \& C. L. QUAVE. 2006. Functional Foods or Food Medicines? On the Consumption of Wild Plants among Albanians and Southern Italians in Lucania. En A. Pieroni \& L. L. Price (eds.), Eating and Healing. Traditional food as medicine, pp. 101-130. Food Products Press-The Haworth Press, New York.

POSEY, D. A. 1983a. Folk Apiculture of the Kayapo Indians of Brazil. Biotropica 15: 154-158.

- 1983b. Keeping of stingless bees by the Kayapo Indians of Brazil. J. Ethnobiology 3: 63-73.

QUEZADA-EUÁN, J. J., W. J. MAY-ITZÁ \& J. A. GONZÁLES-ACERETO. 2001. Meliponiculture in Mexico: problems and perspective for development. Bee World 82: 160-167. 
RAMOS-ELORDUY, J.\& M. PINO. 2003. Enfermedades tratadas con insectos en el Continente Americano. Entomol. Mex. 2: 604-611.

RÍOS RUIZ, S. \& V. MARTÍNEZ FRANCÉS. 2003. Plantas de los Herberos en la Sierra Mariola (SW de Valencia, N-NW de Alicante, España). Flora Montiberica 25: 42-51.

RODRIGUES, D.S. 2005. Etnoconhecimento sobre abellas sem ferrão: saberes e práticas dos índios Guarani m’bya na mata atlántica. Tesis Maestría. Universidad de São Paulo, Piracicaba. 237 pp.

SCARPA, G. F. 2004. Medicinal plants used by the Criollos of Northwestern Argentine Chaco. J. Ethnopharmacol. 91:115-135.

SCHWARZ, H. F. 1948. Stingless bees (Meliponidae) of the Western Hemisphere. Bull. Am. Mus. Nat. Hist. 90: 1-546.

SILVEIRA, F. A., G. A. R. MELO \& E. A. B. ALMEIDA. 2002. Abelhas Brasileiras. Sistemática e Identificação, Belo Horizonte. 253 pp.

SUBRAMANIAN, R., H. UMESH HEBBAR \& N. K. RASTOGI. 2007. Processing of honey: a review.
International Journal of Food Properties 10: 127-143.

VANDEBROEK, M. J., A. BALICK, F. OSOSKI, J. KRONENBERG, C. YUKES, F. WADE, B. P. JIMÉNEZ \& D. CASTILLO. 2010. The importance of botellas and other plant mixtures in Dominican traditional medicine. J.Ethnopharmacol. 128: 2041.

VIT, P., M. MEDINA \& M. E. ENRIQUEZ. 2004. Quality standards for medicinal uses of Meliponinae honey in Guatemala, Mexico and Venezuela. Bee World 85: 2-5.

ZAMUDIO, F., M. KUJAWSKA \& N. I. HILGERT. 2010. Honey as Medicinal and Food Resource. Comparison between Polish and Multiethnic Settlements of the Atlantic Forest, Misiones, Argentina. The Open Complementary Medicine Journal 2: 58-73.

\& N. I. HILGERT. 2011. ¿Cómo los conocimientos locales aportan información sobre la riqueza de especies de abejas sin aguijón (Apidae: Meliponini) del norte de Misiones, Argentina? JAN 2012, VOL. $37 \mathrm{~N}^{\mathrm{o}} 1$ Interciencia. 37: en prensa.

Original recibido el 8 de septiembre de 2011; aceptado el 21 de noviembre de 2011. 